appropriateness of including our trial in the same group as the other randomized glutamine trials performed in the critical care setting, given differences in patient population, administration route, dosage, and approach to feeding.

Buijs et al. express several concerns that we believe are overstated. First, we attempted to optimize nutrition delivery through the application of evidence-informed strategies. Our success with feeding is probably consistent with the results in most intensive care units worldwide. The overall degree of imbalance in baseline characteristics is consistent with expected random variation and did not affect our conclusions. Our prespecified analysis plan, which followed best practices for factorial, randomized, controlled trials, did not adjust for the number of organ failures..$^{2-5}$ Nevertheless, when we adjust for important baseline characteristics (including organ failures), the adjusted odds ratio of 28-day mortality for glutamine as compared with placebo remains consistent with our primary analysis, at 1.4 (95\% confidence interval, 1.0 to $2.1 ; \mathrm{P}=0.05)$. Finally, our secondary 6-month mortality results were based on survival analysis techniques that remain valid in the presence of random loss to follow-up. Besides, almost the entire treatment effect was observed within the first 30 days, when there was almost no loss to follow-up.

Daren Heyland, M.D.

Kingston General Hospital

Kingston, ON, Canada

dkh2@queensu.ca

Paul E. Wischmeyer, M.D.

University of Colorado

Aurora, CO

Andrew G. Day, M.Sc.

Kingston General Hospital

Kingston, ON, Canada

for the Canadian Clinical Care Trials Group

Since publication of their article, the authors report no further potential conflict of interest.

1. The 2011 Oxford CEBM levels of evidence. Oxford, United Kingdom: Oxford Centre for Evidence-Based Medicine, 2013 (http://www.cebm.net/index.aspx?o=5653).

2. Heyland DK, Dhaliwal R, Day AG, et al. REducing Deaths due to OXidative Stress (the REDOXS Study): rationale and study design for a randomized trial of glutamine and antioxidant supplementation in critically ill patients. Proc Nutr Soc 2006; 65:250-63.

3. Raab GM, Day S, Sales J. How to select covariates to include in the analysis of a clinical trial. Control Clin Trials 2000;21:33042.

4. McAlister FA, Straus SE, Sackett DL, Altman DG. Analyzing and reporting of factorial trials: a systematic review. JAMA 2003; 289:2545-53.

5. Montgomery AA, Peters TJ, Little P. Design, analysis, and presentation of factorial randomised controlled trials. BMC Med Res Methodol 2003;3:26.

DOI: 10.1056/NEJMc1306658

\title{
Weight Loss in Persons with Serious Mental Illness
}

TO THE EDITOR: Daumit and colleagues (April 25 issue ${ }^{1}$ describe the results of the Randomized Trial of Achieving Healthy Lifestyles in Psychiatric Rehabilitation (ACHIEVE), and they report that an intensive behavioral weight-loss intervention significantly reduced weight in adults with severe mental illness. This comprehensive intervention used existing rehabilitation-program staff in community-based psychiatric rehabilitation settings.

Unfortunately, the 2011 Centers for Medicare and Medicaid Services (CMS) policy for weightloss counseling ${ }^{2}$ would not cover this effective intervention. The CMS policy covers only brief weight-loss counseling delivered by primary care physicians in primary care settings, not the comprehensive counseling by behavioral providers in a rehabilitation setting that Daumit and colleagues found effective. Current CMS policy will have little effect on health disparities because populations that are disproportionately affected by obesity often use the services of community-based organizations. Further, unlike the offices of most primary care doctors, community clinics employ behavioral counselors and typically have facilities (e.g., kitchens and recreation rooms) that are conducive to behavioral weight-loss programs.

The study by Daumit et al. and other community-based obesity trials ${ }^{3}$ provide support for the case for CMS to cover a wider range of settings and providers. This is not only consistent with the evidence but is also the most feasible approach to reducing weight in underserved populations. 
Sherry Pagoto, Ph.D.

Stephenie Lemon, Ph.D.

University of Massachusetts Medical School

Worcester, MA

sherry.pagoto@umassmed.edu

Jessica Whiteley, Ph.D.

University of Massachusetts Boston

Boston, MA

for the Society of Behavioral Medicine

No potential conflict of interest relevant to this letter was reported.

1. Daumit GL, Dickerson FB, Wang NY, et al. A behavioral weight-loss intervention in persons with serious mental illness. N Engl J Med 2013;368:1594-602.

2. Centers for Medicare and Medicaid Services. Decision memo for intensive behavioral therapy for obesity. 2011 (http://www .cms.gov/medicare-coverage-database/details/nca-decision-memo .aspx?\&NcaName=Intensive\%20Behavioral\%20Therapy\%20for\% 200besity\&bc $=$ ACAAAAAAIAAA $\&$ NCAId $=253 \&$ ).

3. Whittemore R. A systematic review of the translational research on the Diabetes Prevention Program. Transl Behav Med 2011;1:480-91.

DOI: 10.1056/NEJMc1306994

TO THE EDITOR: Daumit et al. bring to light an important and often overlooked dynamic of treating patients with mental illness. Since one third of the U.S. population is classified as obese, and mentally ill patients are at increased risk for being overweight, it is critical that all health care professionals, specifically those working within the mental health field, integrate within the patient-physician relationship a discussion regarding healthy eating and lifestyle habits.

As mental health care providers struggle to do more with less funding, this study exemplifies what can be accomplished with relatively low overhead. The seamless integration of the ACHIEVE protocol into outpatient mental health centers and the use of the already established infrastructure within these centers increase the validity of this study.

Future studies of the effect of this behavioral intervention on coexisting conditions such as cardiovascular disease, diabetes, and dyslipidemia may reinforce the importance of addressing the benefits of a healthy lifestyle with patients who have mental illness.

Joshua R. Ackerman, M.D.

New York Medical College

New York, NY

No potential conflict of interest relevant to this letter was reported.
THE AUTHORS REPLY: The implementation and dissemination of effective lifestyle interventions are critical to stem the epidemic of obesity and obesity-related conditions in persons with serious mental illness. However, incorporating weight-loss interventions into behavioral health settings will require financial and organizational resources that are unavailable in most community mental health settings. Community mental health programs are often under financial stress, and mental health, not physical health, is their first priority.

As Pagoto et al. state, the current CMS policy of providing reimbursement for weight counseling by primary care physicians would not be applicable to the ACHIEVE intervention, which was delivered by health coaches and staff. However, the federal government does provide two potential sources of funding for health promotion to organizations that serve persons with serious mental illness. The Patient Protection and Affordable Care Act (ACA) "health home" provision gives states additional federal funding for care management and the use of clinical information systems by Medicaid providers to enhance coordination of medical and behavioral health care. ${ }^{1}$ Eligible Medicaid recipients must have a serious and persistent mental health condition, two or more chronic mental or physical health conditions, or one mental or physical condition with a high risk of another. Primarily through ACA funding, the Substance Abuse and Mental Health Services Administration has awarded more than 60 grants to communitybased agencies with the goal of building infrastructure to support integration of primary care services for persons with serious mental illness. ${ }^{2}$ These initiatives do not explicitly fund lifestyle interventions, although program resources could be used for incorporating health promotion into mental health care. Still, these two initiatives are modest in scope. We concur with Pagoto and colleagues that broader coverage of weightmanagement counseling is needed.

We also agree with Ackerman that incorporating healthy-lifestyle interventions into community mental health programs, particularly those such as psychiatric rehabilitation programs that mental health consumers attend frequently, is a wonderful but unrealized opportunity to leverage the existing infrastructure. 
Weight-loss interventions could be incorporated into regular programming. In any case, implementing the ACHIEVE intervention into a broader array of community mental health settings would require resources to ensure the effective translation of our findings into practice.

Gail L. Daumit, M.D., M.H.S.

Johns Hopkins Medical Institutions

Baltimore, MD

gdaumit@jhmi.edu

Faith B. Dickerson, Ph.D., M.P.H.

Sheppard Pratt Health System

Baltimore, MD
Lawrence J. Appel, M.D., M.P.H.

Johns Hopkins Medical Institutions

Baltimore, MD

Since publication of their article, the authors report no further potential conflict of interest.

1. Health homes for enrollees with chronic conditions. Document 10-024. Baltimore: Center for Medicare and Medicaid Services, November 16, 2010 (http://downloads.cms.gov/cmsgov/ archived-downloads/SMDL/downloads/SMD10024.pdf).

2. Primary and behavioral health integration grants. Rockville, MD: Substance Abuse and Mental Health Services Administration (http://www.samhsa.gov/healthreform/healthhomes).

\section{Darbepoetin Alfa in Systolic Heart Failure}

TO THE EDITOR: In their report on the Reduction of Events by Darbepoetin Alfa in Heart Failure (RED-HF) trial, Swedberg et al. (March 28 issue) ${ }^{1}$ conclude that the correction of anemia with the use of darbepoetin alfa in patients with heart failure does not reduce the rate of cardiovascular end points. This finding is in line with the results of the Trial to Reduce Cardiovascular Events with Aranesp Therapy (TREAT). ${ }^{2}$ However, it may be difficult to generalize such results to all forms of anemia in heart failure, especially to anemia caused by iron deficiency. As found by Toblli et al. ${ }^{3}$ and also shown in the Ferinject Assessment in Patients with Iron Deficiency and Chronic Heart Failure (FAIR-HF) trial, ${ }^{4}$ iron supplementation in patients with heart failure and iron deficiency reduces $\mathrm{N}$-terminal pro-brain natriuretic peptide levels and improves symptoms and functional capacity. Since the FAIR-HF trial did not evaluate objective end points, the follow-up Iron in Congestive Heart Failure (ICHF) trial (ClinicalTrials.gov number, NCT01837082) will evaluate the effect of iron supplementation on left ventricular ejection fraction and renal function with the use of magnetic resonance imaging of the heart and radionucleotide measurement.

It seems plausible to assume that taking into account the patient's iron status in the therapeutic regimen may be superior to an approach focusing only on the hemoglobin level. Therefore, it is important that the authors stratify the primary end point according to the iron status at baseline, especially since iron deficiency was rigorously corrected during the trial.

\section{Mahir Karakas, M.D. Wolfgang Koenig, M.D.}

University of Ulm Medical Center Ulm, Germany

mahir.karakas@uniklinik-ulm.de

No potential conflict of interest relevant to this letter was reported.

1. Swedberg K, Young JB, Anand IS, et al. Treatment of anemia with darbepoetin alfa in systolic heart failure. $\mathrm{N}$ Engl J Med 2013;368:1210-9.

2. Pfeffer MA, Burdmann EA, Chen C-Y, et al. A trial of darbepoetin alfa in type 2 diabetes and chronic kidney disease. $N$ Engl J Med 2009;361:2019-32.

3. Toblli JE, Lombrana A, Duarte P, Di Gennaro F. Intravenous iron reduces NT-pro-brain natriuretic peptide in anemic patients with chronic heart failure and renal insufficiency. J Am Coll Cardiol 2007;50:1657-65.

4. Anker SD, Colet JC, Filippatos G, et al. Ferric carboxymaltose in patients with heart failure and iron deficiency. $\mathrm{N}$ Engl J Med 2009;361:2436-48.

DOI: $10.1056 / N E J M c 1306321$

TO THE EDITOR: The RED-HF investigators found that darbepoetin alfa had no effect in patients with anemia and heart failure. But was the untreated group really untreated? Almost all the patients came into the program with a normal transferrin saturation of more than $20 \%$; indeed, the median level was $24 \%$ (interquartile range, 19 to 31). This percentage is much above what is typically found in heart failure, in which the majority of patients have a transferrin saturation of less than $20 \%$, suggesting iron deficiency. ${ }^{1-3}$ Thus, presumably the iron deficiency was corrected before they entered the study. In addition, once enrolled in the program, the great majority of patients received intravenous iron, oral iron, 